The Journal of $\mathbf{N}_{\text {onlinear }} \mathbf{S}_{\text {cience and }}$ Applications http://www.tjnsa.com

\title{
ON A CERTAIN CLASS OF HARMONIC MULTIVALENT FUNCTIONS
}

\author{
SAURABH PORWAL ${ }^{1}$, POONAM DIXIT $^{2, *}$ AND VINOD KUMAR ${ }^{3}$ \\ Dedicated to Themistocles M. Rassias on the occasion of his sixtieth birthday \\ Communicated by Choonkil Park
}

\begin{abstract}
The purpose of the present paper is to study some results involving coefficient conditions, extreme points, distortion bounds, convolution conditions and convex combination for a new class of harmonic multivalent functions in the open unit disc. Relevant connections of the results presented here with various known results are briefly indicated.
\end{abstract}

\section{INTRODUCTION}

A continuous complex-valued function $f=u+i v$ defined in a simply connected domain $D$ is said to be harmonic in $D$ if both $u$ and $v$ are real harmonic in $D$. In any simply connected domain we can write $f=h+\bar{g}$, where $h$ and $g$ are analytic in $D$. We call $h$ the analytic part and $g$ the co-analytic part of $f$.

A necessary and sufficient condition for $f$ to be locally univalent and sensepreserving in $D$ is that $\left|h^{\prime}(z)\right|>\left|g^{\prime}(z)\right|, z \in D$. See Clunie and Shiel-Small [7], (see also Ahuja [1] and Duren [11].)

Let $H$ denotes the class of functions $f=h+\bar{g}$ which are harmonic univalent and sense-preserving in the open unit disk $U=\{z:|z|<1\}$ for which $f(0)=$ $f_{z}(0)-1=0$. Then for $f=h+\bar{g} \in S_{H}$ we may express the analytic functions $h$ and $g$ as

$$
h(z)=z+\sum_{k=2}^{\infty} a_{k} z^{k}, g(z)=\sum_{k=1}^{\infty} b_{k} z^{k},\left|b_{1}\right|<1 .
$$

Date: Received: August 27, 2010; Revised: November 29, 2010.

*Corresponding author

(c) 2011 N.A.G.

2000 Mathematics Subject Classification. Primary 30C45, 30C50, 30C55.

Key words and phrases. Harmonic; Univalent; Multivalent functions; Fractional calculus. 
Recently, Ahuja and Jahangiri [3] defined the class

$$
H_{p}(k),(p, k \in N=\{1,2,3 \ldots\})
$$

consisting of all p-valent harmonic functions $f=h+\bar{g}$ which are sense-preserving in $U$ and $h$ and $g$ are of the form

$$
h(z)=z^{p}+\sum_{k=2}^{\infty} a_{k+p-1} z^{k+p-1}, g(z)=\sum_{k=1}^{\infty} b_{k+p-1} z^{k+p-1}, \quad\left|b_{p}\right|<1 .
$$

Note that $H$ and $H_{p}(k)$ reduce to the class $S$ and $S_{p}(k)$ of analytic univalent and multivalent functions, respectively, if the co-analytic part of its members are zero. For these classes $f(z)$ may be expressed as

$$
f(z)=z+\sum_{k=2}^{\infty} a_{k} z^{k}
$$

and

$$
f(z)=z^{p}+\sum_{k=2}^{\infty} a_{k+p-1} z^{k+p-1}
$$

The following definitions of fractional integrals and fractional derivatives are due to Owa [17] and Srivastava and Owa [23].

Definition 1.1. The fractional integral of order $\lambda$ is defined for a function $f(z)$ by

$$
D_{z}^{-\lambda} f(z)=\frac{1}{\Gamma \lambda} \int_{0}^{z} \frac{f(\varsigma)}{(z-\varsigma)^{1-\lambda}} d \varsigma
$$

where $\lambda>0, f(z)$ is an analytic function in a simply connected region of the $z$-plane containing the origin and the multiplicity of $(z-\varsigma)^{\lambda-1}$ is removed by requiring $\log (z-\varsigma)$ to be real when $(z-\varsigma)>0$.

Definition 1.2. The fractional derivative of order $\lambda$ is defined for a function $f(z)$ by

$$
D_{z}^{\lambda} f(z)=\frac{1}{\Gamma(1-\lambda)} \frac{d}{d z} \int_{0}^{z} \frac{f(\varsigma)}{(z-\varsigma)^{\lambda}} d \varsigma,
$$

where $0 \leq \lambda<1, f(z)$ is an analytic function in a simply connected region of the $z$-plane containing the origin and the multiplicity of $(z-\varsigma)^{-\lambda}$ is removed as in Definition 1.1 above.

Definition 1.3. Under the hypothesis of Definition 1.2 the fractional derivative of order $n+\lambda$ is defined for a function $f(z)$ by

$$
D_{z}^{n+\lambda} f(z)=\frac{d^{n}}{d z^{n}} D_{z}^{\lambda} f(z)
$$

where $0 \leq \lambda<1$ and $n \in N_{0}=\{0,1,2 \ldots$.$\} .$ 
Motivated with the definition of Salagean operator, we introduced an interesting operator $\left(D_{z}^{\lambda, p}\right)^{n} f(z)$ for function $f(z)$ of the form (1.4)

$$
\begin{aligned}
\left(D_{z}^{\lambda, p}\right)^{0} f(z) & =\frac{\Gamma(p-\lambda+1)}{\Gamma(p+1)} z^{\lambda} D_{z}^{\lambda} f(z) \\
\left(D_{z}^{\lambda, p}\right)^{1} f(z) & =\frac{z}{p}\left(\frac{\Gamma(p-\lambda+1)}{\Gamma(p+1)} z^{\lambda} D_{z}^{\lambda} f(z)\right)^{\prime} \\
\left(D_{z}^{\lambda, p}\right)^{n} f(z) & =\left(D_{z}^{\lambda, p}\right)\left(\left(D_{z}^{\lambda, p}\right)^{n-1} f(z)\right) .
\end{aligned}
$$

Thus we have

$$
\begin{aligned}
\left(D_{z}^{\lambda, p}\right)^{n} f(z) & =z^{p}+\sum_{k=2}^{\infty}\left(\frac{(k+p-1) \Gamma(p-\lambda+1) \Gamma(k+p)}{p \Gamma(p+1) \Gamma(k+p-\lambda)}\right)^{n} a_{k+p-1} z^{k+p-1} \\
& =z^{p}+\sum_{k=2}^{\infty}(\varphi(k, p, \lambda))^{n} a_{k+p-1} z^{k+p-1}
\end{aligned}
$$

where and throughout this paper

$$
\varphi(k, p, \lambda)=\frac{k \Gamma(p-\lambda+1) \Gamma(k+p)}{\Gamma(p+1) \Gamma(k+p-\lambda)}(k, p \in N) .
$$

Now, we define $\left(D_{z}^{\lambda, p}\right)^{n} f(z)$ for function of the form (1.2) as follows

$$
\left(D_{z}^{\lambda, p}\right)^{n} f(z)=\left(D_{z}^{\lambda, p}\right)^{n} h(z)+(-1)^{n} \overline{\left(D_{z}^{\lambda, p}\right)^{n} g(z)} .
$$

We note that the study of the above operator $\left(D_{z}^{\lambda, p}\right)^{n}$ is of special interest because it includes a variety of well-known operator. For example

1. If we put $n=0, p=1$ then it reduces to Owa-Srivastava Operator.

2. If we put $\lambda=0$, then it reduces to well-known and widely used Salagean Operator [19].

Now for

$$
m \in N, n \in N_{0}, m>n, 0 \leq \gamma<1, \beta \geq 0,0 \leq \lambda<1,0 \leq t \leq 1, \alpha \in R
$$

and $z \in U$, suppose that $H_{p}(m, n ; \beta ; \gamma ; t ; \lambda)$ denote the family of harmonic functions $f$ of the form (1.2) such that

$$
\operatorname{Re}\left\{\left(1+\beta e^{i \alpha}\right) \frac{\left(D_{z}^{\lambda, p}\right)^{m} f(z)}{\left(D_{z}^{\lambda, p}\right)^{n} f_{t}(z)}-\beta e^{i \alpha}\right\} \geq \gamma
$$

where $\left(D_{z}^{\lambda, p}\right)^{m} f(z)$ is defined by $(1.10)$ and $f_{t}(z)=(1-t) z+t(h(z)+\overline{g(z)})$.

Further, let the subclass $\overline{H_{p}}(m, n ; \beta ; \gamma ; t ; \lambda)$ consisting of harmonic functions $f_{m}=h+\overline{g_{m}}$ in $H_{p}(m, n ; \beta ; \gamma ; t ; \lambda)$ so that $h$ and $g_{m}$ are of the form

$$
h(z)=z^{p}-\sum_{k=2}^{\infty}\left|a_{k+p-1}\right| z^{k+p-1}, g_{m}(z)=(-1)^{m-1} \sum_{k=1}^{\infty}\left|b_{k+p-1}\right| z^{k+p-1}, \quad\left|b_{p}\right|<1 .
$$

By specializing the parameters in subclass $H_{p}(m, n ; \beta ; \gamma ; t ; \lambda)$, we obtain the following known subclasses studied earlier by various authors.

(1) $H_{p}(m, n ; 0 ; \gamma ; 1 ; 0) \equiv H_{p}(m, n ; \gamma)$ and $\overline{H_{p}}(m, n ; 0 ; \gamma ; 1 ; 0) \equiv \overline{H_{p}}(m, n ; \gamma)$ studied by Sker and Eker [20]. 
(2) $H_{p}(n+1, n ; 1 ; \gamma ; 1 ; 0) \equiv H_{p}(n, \gamma)$ and $\overline{H_{p}}(n+1, n ; 1 ; \gamma ; 1 ; 0) \equiv \overline{H_{p}}(n, \gamma)$ studied by Dixit et al. [9].

(3) $H_{p}(1,0 ; 0 ; \gamma ; 1 ; 0) \equiv H_{p}(\gamma)$ and $\overline{H_{p}}(1,0 ; 0 ; \gamma ; 1 ; 0) \equiv \overline{H_{p}}(\gamma)$ studied by Ahuja and Jahangiri [3].

(4) $H_{1}(n+q, n, p, \gamma, 1,0) \equiv R_{H}(n, \gamma, p, q)$ studied by Dixit et al. [8].

(5) $H_{1}(n+1, n, 1, \gamma, 1,0) \equiv R S_{H}(n, \gamma)$ and $\overline{H_{1}}(n+1, n, 1, \gamma, 1,0) \equiv \overline{R S_{H}}(n, \gamma)$ studied by Yalcin et al. [25].

(6) $H_{1}(1,0,1, \gamma, 1,0) \equiv G_{H}(\gamma)$ and $\overline{H_{1}}(1,0,1, \gamma, 1,0) \equiv \overline{G_{H}}(\gamma)$ studied by Rosy et al. [18].

(7) $H_{1}(2,1, \beta, \gamma, 1,0) \equiv H C V(\beta, \gamma)$ and $\overline{H_{1}}(2,1, \beta, \gamma, 1,0) \equiv \overline{H C V}(\beta, \gamma)$ studied by Kim et al. [15].

(8) $H_{1}(1,0, \beta, \gamma, t, 0) \equiv G_{H}(\beta, \gamma, t)$ and $\overline{H_{1}}(1,0, \beta, \gamma, t, 0) \equiv \overline{G_{H}}(\beta, \gamma, t)$ studied by Ahuja et al. [2].

(9) $H_{1}(m, n ; 0 ; \gamma ; 1 ; 0) \equiv S_{H}(m, n ; \gamma)$ and $\overline{H_{1}}(m, n ; 0 ; \gamma ; 1 ; 0) \equiv \overline{S_{H}}(m, n ; \gamma)$ studied by Yalcin [24].

(10) $H_{1}(n+1, n ; 0 ; \gamma ; 1 ; 0) \equiv H(n, \gamma)$ and $\overline{H_{1}}(n+1, n ; 0 ; \gamma ; 1 ; 0) \equiv \bar{H}(n, \gamma)$ studied by Jahangiri et al. [13].

(11) $H_{1}(1,0 ; 0 ; \gamma ; t ; \lambda) \equiv S_{H}^{*}(\gamma, t, \lambda)$ and $\overline{H_{1}}(1,0 ; 0 ; \gamma ; t ; \lambda) \equiv \overline{S_{H}^{*}}(\gamma, t, \lambda)$ studied by Kumar et al. [16].

(12) $H_{1}(1,0 ; 0 ; \gamma ; 1 ; \lambda) \equiv S_{H}^{*}(\gamma, \lambda)$ and $\overline{H_{1}}(1,0 ; 0 ; \gamma ; 1 ; \lambda) \equiv T S_{H}^{*}(\gamma, \lambda)$ studied by Dixit and Porwal [10].

(13) $H_{1}(2,1 ; 0 ; \gamma ; 1 ; 0) \equiv H K(\gamma)$ and $H_{1}(1,0 ; 0 ; \gamma ; 1 ; 0) \equiv S_{H}^{*}(\gamma)$ studied by Jahangiri [12].

(14) $H_{1}(2,1 ; 0 ; 0 ; 1 ; 0) \equiv H K(0)$ and $H_{1}(2,1 ; 0 ; 0 ; 1 ; 0) \equiv S_{H}^{*}(0)$ studied by Avci and Zlotkiewicz [6], Silverman [21] and Silverman and Silvia [22].

(15) $H_{1}(1,0,0, \gamma, 0,0) \equiv N_{H}(\gamma)$ studied by Ahuja and Jahangiri [4].

(16) $S_{p}(1,0, k, 0,1,0) \equiv k-S T$ studied by Aouf [5], see also Kanas and Srivastava [14].

In the present paper, results involving the coefficient condition, extreme points, distortion bounds, convolution and convex combinations for the above classes $H_{p}(m, n, \beta, \gamma, t \lambda)$ and $\overline{H_{p}}(m, n, \beta, \gamma, t, \lambda)$ of harmonic multivalent functions have been investigated.

\section{MAIN RESULTS}

In our first theorem, we introduce a sufficient condition for functions in $H_{p}(m, n, \beta, \gamma, t, \lambda)$.

Theorem 2.1. Let $f=h+\bar{g}$ be such that $h$ and $g$ are given by (1.2). Furthermore, let

$$
\sum_{k=1}^{\infty}\left\{\psi(m, n, \beta, \gamma, t, \lambda)\left|a_{k+p-1}\right|+\Theta(m, n, \beta, \gamma, t, \lambda)\left|b_{k+p-1}\right|\right\} \leq 2,
$$

where

$$
\psi(m, n, \beta, \gamma, t, \lambda)=\frac{(\varphi(k, p, \lambda))^{m}(1+\beta)-t(\beta+\gamma)(\varphi(k, p, \lambda))^{n}}{1-\gamma}
$$


and

$$
\Theta(m, n, \beta, \gamma, t, \lambda)=\frac{(\varphi(k, p, \lambda))^{m}(1+\beta)-(-1)^{m-n} t(\beta+\gamma)(\varphi(k, p, \lambda))^{n}}{1-\gamma},
$$

$a_{p}=1, m \in N, n \in N_{0}, m>n, 0 \leq \gamma<1, \beta \geq 0,0 \leq \lambda<1$ and $0 \leq t \leq 1$, then $f$ is sense-preserving in $U$ and $f \in H_{p}(m, n, \beta, \gamma, t, \lambda)$.

Proof. Using the fact that Re $\omega \geq \gamma$ if and only if $|1-\gamma+\omega| \geq|1+\gamma-\omega|$, it suffices to show that

$$
\begin{array}{r}
\left|(1-\gamma)\left(D_{z}^{\lambda, p}\right)^{n} f_{t}(z)+\left(1+\beta e^{i \alpha}\right)\left(D_{z}^{\lambda, p}\right)^{m} f(z)-\beta e^{i \alpha}\left(D_{z}^{\lambda, p}\right)^{n} f_{t}(z)\right| \\
-\left|(1+\gamma)\left(D_{z}^{\lambda, p}\right)^{n} f_{t}(z)-\left(1+\beta e^{i \alpha}\right)\left(D_{z}^{\lambda, p}\right)^{m} f(z)+\beta e^{i \alpha}\left(D_{z}^{\lambda, p}\right)^{n} f_{t}(z)\right| .
\end{array}
$$

Substituting for $\left(D_{z}^{\lambda, p}\right)^{m} f(z)$ and $\left(D_{z}^{\lambda, p}\right)^{n} f_{t}(z)$ in L.H.S. of $(2.2)$, we have

$$
\begin{aligned}
& \mid(2-\gamma) z^{p}+\sum_{k=2}^{\infty}\left[\left(1-\gamma-\beta e^{i \alpha}\right)(\varphi(k, p, \lambda))^{n} t+\left(1+\beta e^{i \alpha}\right)(\varphi(k, p, \lambda))^{m}\right] a_{k+p-1} z^{k+p-1} \\
& +(-1)^{n} \sum_{k=1}^{\infty}\left[\left(1-\gamma-\beta e^{i \alpha}\right)(\varphi(k, p, \lambda))^{n} t+(-1)^{m-n}\left(1+\beta e^{i \alpha}\right)(\varphi(k, p, \lambda))^{m}\right] \bar{b}_{k+p-1} \bar{z}^{k+p-1} \\
& -\mid \gamma z^{p}+\sum_{k=2}^{\infty}\left[\left(1+\gamma+\beta e^{i \alpha}\right)(\varphi(k, p, \lambda))^{n} t-\left(1+\beta e^{i \alpha}\right)(\varphi(k, p, \lambda))^{m}\right] a_{k+p-1} z^{k+p-1} \\
& +(-1)^{n} \sum_{k=1}^{\infty}\left[\left(1+\gamma+\beta e^{i \alpha}\right)(\varphi(k, p, \lambda))^{n} t-(-1)^{m-n}\left(1+\beta e^{i \alpha}\right)(\varphi(k, p, \lambda))^{m}\right] \bar{b}_{k+p-1} \bar{z}^{k+p-1} \mid \\
& \geq 2(1-\gamma)|z|^{p}-2 \sum_{k=2}^{\infty}\left[(\varphi(k, p, \lambda))^{m}(1+\beta)-(\gamma+\beta) t(\varphi(k, p, \lambda))^{n}\right]\left|a_{k+p-1}\right||z|^{k+p-1} \\
& -\sum_{k=1}^{\infty}\left|\left[(1-\gamma)(\varphi(k, p, \lambda))^{n} t+(-1)^{m-n}\left(1+\beta e^{i \alpha}\right)(\varphi(k, p, \lambda))^{m}-\beta e^{i \alpha}(\varphi(k, p, \lambda))^{n} t\right]\right|\left|b_{k+p-1}\right||z|^{k+p-1} \\
& -\sum_{k=1}^{\infty}\left|\left[(-1)^{m-n}\left(1+\beta e^{i \alpha}\right)(\varphi(k, p, \lambda))^{m}-\left(1+\gamma+\beta e^{i \alpha}\right)(\varphi(k, p, \lambda))^{n} t\right]\right|\left|b_{k+p-1}\right||z|^{k+p-1} \\
& =\left\{\begin{array}{l}
2(1-\gamma)|z|^{p}-2 \sum_{k=2}^{\infty}\left[(1+\beta)(\varphi(k, p, \lambda))^{m}-(\gamma+\beta) t(\varphi(k, p, \lambda))^{n}\right]\left|a_{k+p-1}\right||z|^{k+p-1} \\
-2 \sum_{k=2}^{\infty}\left[(1+\beta)(\varphi(k, p, \lambda))^{m}+(\gamma+\beta) t(\varphi(k, p, \lambda))^{n}\right]\left|b_{k+p-1}\right||z|^{k+p-1}, \text { if } m-n \text { is odd } \\
2(1-\gamma)|z|^{p}-2 \sum_{k=2}^{\infty}\left[(1+\beta)(\varphi(k, p, \lambda))^{m}-(\gamma+\beta) t(\varphi(k, p, \lambda))^{n}\right]\left|a_{k+p-1}\right||z|^{k+p-1} \\
-2 \sum_{k=1}^{\infty}\left[(1+\beta)(\varphi(k, p, \lambda))^{m}-(\gamma+\beta) t(\varphi(k, p, \lambda))^{n}\right]\left|b_{k+p-1}\right||z|^{k+p-1}, \text { if } m-n \text { is odd }
\end{array}\right.
\end{aligned}
$$




$$
\begin{aligned}
& =2(1-\gamma)|z|^{p}\left\{1-\sum_{k=2}^{\infty} \frac{(\varphi(k, p, \lambda))^{m}(1+\beta)-(\gamma+\beta) t(\varphi(k, p, \lambda))^{n}}{1-\gamma}\left|a_{k+p-1}\right||z|^{k-1}\right. \\
& \left.-\sum_{k=1}^{\infty} \frac{(\varphi(k, p, \lambda))^{m}(1+\beta)-(-1)^{m-n}(\gamma+\beta) t(\varphi(k, p, \lambda))^{n}}{1-\gamma}\left|b_{k+p-1}\right||z|^{k-1}\right\} \\
& >2(1-\gamma)\left\{1-\sum_{k=2}^{\infty} \frac{(\varphi(k, p, \lambda))^{m}(1+\beta)-(\gamma+\beta) t(\varphi(k, p, \lambda))^{n}}{1-\gamma}\left|a_{k+p-1}\right|\right. \\
& \left.-\sum_{k=1}^{\infty} \frac{(\varphi(k, p, \lambda))^{m}(1+\beta)-(-1)^{m-n}(\gamma+\beta) t(\varphi(k, p, \lambda))^{n}}{1-\gamma}\left|b_{k+p-1}\right|\right\} \text {. }
\end{aligned}
$$

The last expression is non negative by (2.1), and so the proof is complete.

In the following theorem, it is proved that the condition (2.1) is also necessary for functions $f_{m}=h+\overline{g_{m}}$, where $\mathrm{h}$ and $g_{m}$ are of the form (1.12).

Theorem 2.2. Let $f_{m}=h+\overline{g_{m}}$ be given by (1.12). Then $f_{m} \in \bar{H}_{p}(m, n, \beta, \gamma, t, \lambda)$, if and only if

$$
\sum_{k=1}^{\infty}\left\{\psi(m, n, \beta, \gamma, t, \lambda)\left|a_{k+p-1}\right|+\Theta(m, n, \beta, \gamma, t, \lambda)\left|b_{k+p-1}\right|\right\} \leq 2 .
$$

Proof. Since $\overline{H_{p}}(m, n ; \beta ; \gamma ; t ; \lambda) \subset H_{p}(m, n ; \beta ; \gamma ; t ; \lambda)$, we only need to prove the "only if" part of the theorem.

To this end, for functions $f_{m}$ of the form (1.12), we notice that condition

$$
\operatorname{Re}\left\{\left(1+\beta e^{i \alpha}\right) \frac{\left(D_{z}^{\lambda, p}\right)^{m} f(z)}{\left(D_{z}^{\lambda, p}\right)^{n} f_{t}(z)}-\beta e^{i \alpha}\right\} \geq \gamma
$$

is equivalent to

$$
\operatorname{Re}\left\{\begin{array}{c}
(1-\gamma) z^{p}-\sum_{k=2}^{\infty}\left[(\varphi(k, p, \lambda))^{m}\left(1+\beta e^{i \alpha}\right)-\left(\beta e^{i \alpha}+\gamma\right)(\varphi(k, p, \lambda))^{n} t\right] \\
\left|a_{k+p-1}\right| z^{k+p-1}+(-1)^{2 m-1} \\
\sum_{k=1}^{\infty}\left[(\varphi(k, p, \lambda))^{m}\left(1+\beta e^{i \alpha}\right)-(-1)^{m-n}\left(\beta e^{i \alpha}+\gamma\right)(\varphi(k, p, \lambda))^{n} t\right] \\
z-\sum_{k=2}^{\infty}(\varphi(k, p, \lambda))^{n} t\left|a_{k+p-1}\right| z^{k+p-1}+(-1)^{m+n-1} \\
\sum_{k=1}^{\infty}(\varphi(k, p, \lambda))^{n} t\left|b_{k+p-1}\right| \bar{z}^{k+p-1}
\end{array}\right\} 0 .
$$


The above required condition (2.4) must hold for all values of $\mathrm{z}$ in $U$. Upon choosing the values of $\mathrm{z}$ on the positive real axis where $0 \leq z=r<1$, we must have

$$
\begin{gathered}
\operatorname{Re}\left\{\begin{array}{c}
(1-\gamma)-\sum_{k=2}^{\infty}\left[(\varphi(k, p, \lambda))^{m}-\gamma t(\varphi(k, p, \lambda))^{n}\right]\left|a_{k+p-1}\right| r^{k-1} \\
\frac{-\sum_{k=1}^{\infty}\left[(\varphi(k, p, \lambda))^{m}-(-1)^{m-n} \gamma t(\varphi(k, p, \lambda))^{n}\right]\left|b_{k+p-1}\right| r^{k-1}}{1-\sum_{k=2}^{\infty}(\varphi(k, p, \lambda))^{n} t\left|a_{k+p-1}\right| r^{k-1}-(-1)^{m-n} \sum_{k=1}^{\infty}(\varphi(k, p, \lambda))^{n} t\left|b_{k+p-1}\right| r^{k-1}} \\
-e^{i \alpha} \frac{-\sum_{k=1}^{\infty} \beta\left((\varphi(k, p, \lambda))^{m}-(-1)^{m-n} t(\varphi(k, p, \lambda))^{n}\right)\left|b_{k+p-1}\right| r^{k-1}}{\left.\sum_{k=2}^{\infty} \beta(\varphi(k, p, \lambda))^{m}-t(\varphi(k, p, \lambda))^{n}\right)\left|a_{k+p-1}\right| r^{k-1}}
\end{array}\right\} \geq 0 .
\end{gathered}
$$

Since $\operatorname{Re}\left(-e^{i \alpha}\right) \geq-\left|e^{i \alpha}\right|=-1$, the above inequality reduces to

$$
\begin{aligned}
& (1-\gamma)-\sum_{k=2}^{\infty}\left[(\varphi(k, p, \lambda))^{m}(1+\beta)-(\beta+\gamma)(\varphi(k, p, \lambda))^{n} t\right]\left|a_{k+p-1}\right| r^{k-1} \\
& \frac{-\sum_{k=1}^{\infty}\left[(\varphi(k, p, \lambda))^{m}(1+\beta)-(-1)^{m-n}(\beta+\gamma)(\varphi(k, p, \lambda))^{n} t\right]\left|b_{k+p-1}\right| r^{k-1}}{1-\sum_{k=2}^{\infty}(\varphi(k, p, \lambda))^{n} t\left|a_{k+p-1}\right| r^{k-1}-(-1)^{m-n} \sum_{k=1}^{\infty}(\varphi(k, p, \lambda))^{n} t\left|b_{k+p-1}\right| r^{k-1}} \geq 0 .
\end{aligned}
$$

If the condition (2.3) does not hold, then the numerator in (2.5) is negative for $\mathrm{r}$ sufficiently close to 1 . Hence there exists a $z_{0}=r_{0}$ in $(0,1)$ for which the quotient in (2.5) is negative. This contradicts the required condition for $f_{m} \in \overline{H_{p}}(m, n ; \beta ; \gamma ; t ; \lambda)$ and so the proof is complete.

We employ the techniques of Dixit et al. [9] in the proofs of Theorems 2.3, 2.4, 2.6 and 2.7 . 
Theorem 2.3. Let $f_{m}$ be given by (1.12). Then $f_{m} \in \overline{H_{p}}(m, n ; \beta ; \gamma ; t ; \lambda)$, if and only if

$$
\begin{gathered}
f_{m}(z)=\sum_{k=1}^{\infty}\left(x_{k+p-1} h_{k+p-1}(z)+y_{k+p-1} g_{m_{k+p-1}}(z)\right), \text { where } h_{p}(z)=z^{p}, \\
h_{k+p-1}(z)=z^{p}-\frac{1}{\psi(m, n, \beta, \gamma, t, \lambda)} z^{k+p-1},(k=2,3,4 \ldots), g_{m_{k+p-1}}(z)=z^{p}+(-1)^{m-1} \\
\frac{1}{\Theta(m, n, \beta, \gamma, t, \lambda)} \bar{z}^{k+p-1},(k=1,2,3 \ldots), x_{k+p-1} \geq 0, \\
y_{k+p-1} \geq 0, \sum_{k=1}^{\infty}\left(x_{k+p-1}+y_{k+p-1}\right)=1 .
\end{gathered}
$$

In particular, the extreme points of $\overline{H_{p}}(m, n ; \beta ; \gamma ; t ; \lambda)$ are $\left\{h_{k+p-1}\right\}$ and $\left\{g_{m_{k+p-1}}\right\}$.

Theorem 2.4. Let $f_{m} \in \overline{H_{p}}(m, n ; \beta ; \gamma ; t ; \lambda)$. Then for $|z|=r<1$ we have

$$
\begin{gathered}
\left|f_{m}(z)\right| \leq\left(1+\left|b_{p}\right|\right) r^{p}+\frac{1}{\left(\frac{(p+1)^{2}}{p(p-\lambda+1)}\right)^{n}} \\
\left.\left(\frac{1-\gamma}{\left(\frac{(p+1)^{2}}{p(p-\lambda+1)}\right)^{m-n}(1+\beta)-t(\gamma+\beta)}-\frac{(1+\beta)-(-1)^{m-n} t(\gamma+\beta)}{\left(\frac{(p+1)^{2}}{p(p-\lambda+1)}\right)^{m-n}(1+\beta)-t(\gamma+\beta)}\left|b_{p}\right|\right) r^{p+1}\right)
\end{gathered}
$$

and

$$
\begin{gathered}
\left|f_{m}(z)\right| \geq\left(1-\left|b_{p}\right|\right) r^{p}-\frac{1}{\left(\frac{(p+1)^{2}}{p(p-\lambda+1)}\right)^{n}} \\
\left(\frac{1-\gamma}{\left(\frac{(p+1)^{2}}{p(p-\lambda+1)}\right)^{m-n}(1+\beta)-t(\gamma+\beta)}-\frac{(1+\beta)-(-1)^{m-n} t(\gamma+\beta)}{\left(\frac{(p+1)^{2}}{p(p-\lambda+1)}\right)^{m-n}(1+\beta)-t(\gamma+\beta)}\left|b_{p}\right|\right) r^{p+1} .
\end{gathered}
$$

The following covering result follows from the left hand inequality in Theorem 2.4 .

Corollary 2.5. Let $f_{m}$ of the form $(1.12)$ be so that $f_{m} \in \overline{H_{p}}(m, n ; \beta ; \gamma ; t ; \lambda)$.

Then

$$
\begin{aligned}
& \left\{\omega:|\omega|<\frac{(\varphi(p, \lambda))^{m}(1+\beta)-(\varphi(p, \lambda))^{n}(\gamma+\beta) t-1+\gamma}{(\varphi(p, \lambda))^{m}(1+\beta)-(\varphi(p, \lambda))^{n}(\gamma+\beta) t}\right. \\
& \left.-\frac{\left((\varphi(p, \lambda))^{m}-1\right)(1+\beta)-t(\gamma+\beta)\left((\varphi(p, \lambda))^{n}-(-1)^{m-n}\right)}{(\varphi(p, \lambda))^{m}(1+\beta)-(\varphi(p, \lambda))^{n}(\gamma+\beta) t}\left|b_{p}\right| \subset f_{m}(U)\right\},
\end{aligned}
$$

where $\phi(p, \lambda)=\left\{\frac{(p+1)^{2}}{p(p-\lambda+1)}\right\}$.

For our next theorem, we need to define the convolution of two harmonic functions. For harmonic functions of the form

$$
f_{m}(z)=z^{p}-\sum_{k=2}^{\infty}\left|a_{k+p-1}\right| z^{k+p-1}+(-1)^{m-1} \sum_{k=1}^{\infty}\left|b_{k+p-1}\right| \bar{z}^{k+p-1}
$$


and

$$
F_{m}(z)=z^{p}-\sum_{k=2}^{\infty}\left|A_{k+p-1}\right| z^{k+p-1}+(-1)^{m-1} \sum_{k=1}^{\infty}\left|B_{k+p-1}\right| \bar{z}^{k+p-1}
$$

we define the convolution of two harmonic functions $f_{m}$ and $F_{m}$ as

$$
\begin{aligned}
\left(f_{m} * F_{m}\right)(z)= & f_{m}(z) * F_{m}(z) \\
= & z^{p}-\sum_{k=2}^{\infty}\left|a_{k+p-1} A_{k+p-1}\right| z^{k+p-1}+(-1)^{m-1} \\
& \sum_{k=1}^{\infty}\left|b_{k+p-1} B_{k+p-1}\right| \bar{z}^{k+p-1} .
\end{aligned}
$$

Using this definition, we show that the class $\overline{H_{p}}(m, n ; \beta ; \gamma ; t ; \lambda)$ is closed under convolution.

Theorem 2.6. For $0 \leq \gamma_{1} \leq \gamma_{2}<1$ let $f_{m} \in \overline{H_{p}}\left(m, n ; \beta ; \gamma_{1} ; t ; \lambda\right)$ and $F_{m} \in$ $\overline{H_{p}}\left(m, n ; \beta ; \gamma_{2} ; t ; \lambda\right)$. Then $f_{m} * F_{m} \in \overline{H_{p}}\left(m, n ; \beta ; \gamma_{2} ; t ; \lambda\right) \subseteq \overline{H_{p}}\left(m, n ; \beta ; \gamma_{1} ; t ; \lambda\right)$.

Next, we show that $\bar{H}_{p}(m, n, \beta, \gamma, t, \lambda)$ is closed under convex combinations of its members.

Theorem 2.7. The class $\overline{H_{p}}(m, n ; \beta ; \gamma ; t ; \lambda)$ is closed under convex combination.

\section{ACKNOWLEDGEMENT}

The authors are thankful to referee for his valuable comments and suggestions.

\section{REFERENCES}

[1] O.P. Ahuja, Planar harmonic univalent and related mappings, J. Inequal. Pure Appl. Math., 6 (4) (2005), Art.122, 1-18.

[2] O.P. Ahuja, R. Aghalary and S.B. Joshi, Harmonic univalent functions associated with $k$-Uniformly starlike functions, J. Math. Res. Sci., 9 (1) (2005), 9-17.

[3] O.P. Ahuja and J.M. Jahangiri, Multivalent harmonic starlike functions, Ann. Univ. Marie Curie-Sklodowska Sect. A, LV1 (2001), 1-13.

[4] O.P. Ahuja and J.M. Jahangiri, Noshiro-type harmonic univalent functions, Sci. Math. Japon., 6 (2) (2002), 253-259.

[5] M.K. Aouf, A generalization of multivalent functions with negative coefficients II, Bull. Korean. Math. Soc., 25 (2) (1998), 221-232.

[6] Y. Avci and E. Zlotkiewicz, On harmonic univalent mappings, Ann. Univ. Mariae CurieSklodowska Sect. A 44 (1990), 1-7.

[7] J. Clunie and T. Sheil-Small, Harmonic univalent functions, Ann. Acad. Sci. Fenn. Ser.AI Math 9 (1984), 3-25.

[8] K. K. Dixit, A. L. Pathak, S. Porwal and R. Agarwal, A new subclass of harmonic univalent functions defined by Salagean operator, Int. J. Contemp. Math. Sci., 4 (8) (2009), 371-383.

[9] K.K. Dixit, A.L. Pathak and R. Tripathi, A new subclass of Goodman-Rønning type harmonic multivalent functions, Proc. Int. Sym. GFTA Malaysia, (2008), 79-94.

[10] K.K. Dixit and Saurabh Porwal, An application of fractional calculus to harmonic univalent functions, Bull. Cal. Math. Soc., 102(4), (2010), 343-352.

[11] P. Duren, Harmonic Mappings in the Plane, Cambridge Tracts in Mathematics, Vol.156, Cambridge University Press, Cambridge,2004, ISBN 0-521-64121-7. 
[12] J.M. Jahangiri, Harmonic functions starlike in the unit disc, J. Math. Anal. Appl. 235, (1999) 470-477.

[13] J.M. Jahangiri, G. Murugusundaramoorthy and K. Vijaya, Salagean-type harmonic univalent functions, Southwest J. Pure Appl. Math., 2 (2002), 77-82.

[14] S. Kanas and H.M. Srivastava, Linear operators associated with $k$-Uniformly convex functions, Integral Transform Spec. Funct., 9 (2) (2000), 121-132.

[15] Y.C. Kim, J.M. Jahangiri and J.H. Choi, Certain convex harmonic functions, Int. J. Math. Math. Sci., 29 (8) (2002), 459-465.

[16] V. Kumar, Saurabh Porwal and Poonam Dixit, A New Subclass of Harmonic Univalent Functions Defined by Fractional Calculus, Ind. J. Math., 52(3), (2010), (Accepted).

[17] S. Owa, On the distortion theorem I, Kyungpook Math. J., 18 (1978), 53-59.

[18] T. Rosy, B.A. Stephen, K.G. Subramanian and J.M. Jahangiri, Goodman-Ronning-type harmonic univalent functions, Kyungpook Math. J., 41 (1) (2001), 45-54.

[19] G.S. Salagean, Subclasses of univalent functions, Complex Analysis-Fifth Romanian Finish Seminar, Bucharest, 1 (1983), 362-372.

[20] Bilal Seker and Sevtap Sümer Eker, On Salagean-type harmonic multivalent functions, General Mathematics, 15(2-3) (2007), 52-63.

[21] H. Silverman, Harmonic univalent functions with negative coefficients, J. Math. Anal. Appl., 220 (1998), 283-289.

[22] H. Silverman and E.M. Silvia, Subclasses of Harmonic univalent functions, New Zealand J. Math., 28 (1999), 275-284.

[23] H.M. Srivastava and S. Owa, An application of the fractional derivative, Math. Japon., 29 (1984), 383-389.

[24] Sibel Yalcin, A new class of Salagean-type harmonic univalent functions, Appl. Math. Lett., 18 (2005), 191-198.

[25] S. Yalcin, M. Öztürk and M. Yamankaradeniz, On the subclass of Salagean-type harmonic univalent functions, J. Inequl. Pure Appl. Math., 8 (2)(2007), Art. 54, 1-17.

${ }^{1}$ Department of Mathematics, Janta College, Bakewar, Etawah (U.P.) India 206124

2,3 Department of Mathematics, Christ Church College, Kanpur (U.P.) India 208001

E-mail address: saurabhjcb@rediffmail.com 